\title{
Proceedings of the winter meeting of the British Neuropsychiatric Association, London, January 1993
}

The topic of the meeting was Cognition in neuropsychiatric disorders.

Dr I Robertson (Cambridge) dealt with the approaches to the assessment and treatment of attentional disorders. He analysed the various components of attention and the disorders resulting from the breakdown of links in this chain. He went on to describe the development of tests based on everyday material such as road maps and television schedules, their predictive value in assessing the outcome of close head injury, and ways in which various attentional deficits could be treated using behavioural techniques.

Dr E Taylor (London) dealt with attention deficit disorder and hyperactivity in children. Recent studies, including his own, have demonstrated that impulsivity and overactivity are diagnostically nonspecific features and that 'attention' tests are only general indices of cognitive impairment. The hyperkinetic syndrome is strongly associated with developmental delays and cognitive impairment. Children with this syndrome perform badly when they have to wait for a stimulus to appear and they prefer an immediate reward to a delayed, larger one. When test conditions are carefully controlled, however, they are able to learn as quickly as normal children. These cognitive deficits are the cause of the abnormal personal relationships and increased psychiatric morbidity observed in these children.

Cognitive aspects of epilepsy were addressed by Drs P Thompson and L Goldstein (London). Dr Thompson commented on the variable prevalence of cognitive difficulties in epileptic patients depending on whether community samples or those attending specialist clinics were studied. She also reviewed the ictal and interictal cognitive abnormalities and the role of cognitive factors in precipitating and controlling seizures. Dr Goldstein compared the cognitive outcome of patients undergoing amygdalohippocampectomy to those who had temporal lobectomy for epilepsy. Psychometric tests yielded very similar results in both groups, but amygdalohippocampectomy tended to be superior, with respect to learning and memory, when the operation was performed on the side mediating the functions tested-for example, leftsided operations for verbal learning. Conversely, temporal lobectomy tended to be superior when the operation was on the contralateral side. Both operations were similar in terms of self reported memory problems. Memory deficits in schizophrenia were discussed by Professor A Baddeley (Cambridge) who considered them to be part of more widespread cognitive deficits. Memory problems are as common in this group as in patients with strokes or moderate to severe head injury attending rehabilitation centres. Schizophrenic patients exhibit impairment of explicit or episodic memory, whereas working memory, implicit and procedural learning are better preserved. There are, however, significant differences from the classical amnesic syndrome, in that semantic memory is also impaired in schizophrenics.

Dr B Toone (London) focused on the cognitive challenge tests in relation to single photon emission CT (SPECT) scanning. In his own study of schizophrenic patients, using a modified version of the Wisconsin card sort test, patients exhibited a significant increase in cerebral blood flow in the dorsolateral prefrontal cortex when compared with controls. This increase was more marked in those who obtained the lowest score on the test and, contrary to previously reported studies, these results argue against structural/organic abnormalities in the dorsolateral prefrontal cortex.

A new way of localising cognitive operations by recording changes in cortical negativity with scalp electrodes was described by Dr C Pleydell-Pearce (Bristol).

The meeting ended with two papers devoted to cognitive deficits in neurological disease. Dr A Feinstein (London) presented his studies of cognitive impairment in multiple sclerosis. He focused on the presence of attention deficits in patients with acute optic neuritis, a common harbinger of multiple sclerosis. These deficits were present only in those who had MRI evidence of cerebral pathology (over half of the patients). A follow up study of patients presenting with clinically isolated syndromes suggested that the deficits initially observed may remain static in those in whom the neurological symptoms do not progress, but more severe cognitive impairment, involving memory functions, became apparent in those who had developed definite multiple sclerosis, especially in those who had entered a chronicprogressive course.

Dr R Brown (London) reviewed the theoretical aspects of cognitive research in Parkinson's disease and discussed future strategies. 